\title{
Discovering Neutrinoless Double beta with NEXT-100
}

\section{F. Monrabal ${ }^{* \dagger}$}

Instituto de Física Corpuscular (IFIC), CSIC \& Universitat de València Calle Catedràtico José Beltrán, 2, 46980 Paterna, Valencia, Spain

E-mail: Erancesc.monrabaldific.uv.es

\section{A. Simon}

Instituto de Física Corpuscular (IFIC), CSIC \& Universitat de València Calle Catedràtico José Beltrán, 2, 46980 Paterna, Valencia, Spain

\section{J.J. Gomez-Cadenas}

Instituto de Física Corpuscular (IFIC), CSIC \& Universitat de València Calle Catedràtico José Beltrán, 2, 46980 Paterna, Valencia, Spain

\begin{abstract}
NEXT is a new experiment to search for neutrinoless double beta decay processes to be operated at the LSC laboratory (Canfranc, Spain). The apparatus is a high pressure gas xenon chamber (HPGXe) filled with $100-150 \mathrm{~kg}$ of gas Xenon enriched at $90 \%$ in the ${ }^{136} \mathrm{Xe}$ isotope. NEXT proposes a novel detection technique called SOFT (Separated Optimized Function TPC) which optimizes both the energy resolution and the measurement of the topological signature of the event. First results from NEXT-DEMO prototype operating at IFIC (Valencia) measures a resolution of $1.92 \% \mathrm{FWHM}$ at $511 \mathrm{keV}$ that extrapolates to $0.87 \% \mathrm{FWHM}$ at $Q_{\beta \beta}$. DEMO prototype also demonstrates the tracking capabilities of a SiPM array near the amplification region. NEXTDEMO results are a full demonstration of the NEXT technology and capabilities.
\end{abstract}

XV Workshop on Neutrino Telescopes,

11-15 November 2013

venice, Italy

\footnotetext{
${ }^{*}$ Speaker.

† on behalf of NEXT Collaboration
} 


\section{Introduction}

We know that neutrinos (a) have mass, (b) their masses are very small compared with the other fermions of the same generation, and (c) are neutral particles that have no protected charges (see for example[(]) and references there in). In the SM neutrinos are massless and all the other fermions are Dirac particles described by a four-component spinor. Neutrinos, on the other hand, could be truly neutral particles, identical to their antiparticles, and could be described as Weyl particles, with only two degrees of freedom. Majorana neutrinos could explain the smallness of neutrino masses (via the see-saw mechanism). They could also be an essential ingredient in leptogenesis theories. If neutrinos are Majorana particles observables related with a lepton number violation are predicted. Of these the best, from the experimental point of view is the detection of a neutrinoless double beta decay [[] []]

The past generations of experiments in the field of the experimental search of the neutrinoless double beta decay have been dominated by solid state detectors (e.g, Germanium calorimeters) enriched in the isotopes with a double beta decay mode. Those detectors display an impressive energy resolution but they show limitations when extrapolating to large masses, including: (a) a relatively large surface background, (b) relatively poor spatial location of candidate events, and (c) high cost.

In the other hand, in the actual generation of experiments new approaches with more capabilities to handle large masses and to reach better level of background suppression have appeared. In this scenario, experiments using ${ }^{136} \mathrm{Xe}$ appear in the field with new techniques and with a competitive sensitivity to the half-life of the process.

\section{NEXT Concept}

The Neutrino Experiment with a Xenon TPC (NEXT) [四] will search for $\beta \beta 0 v$ in ${ }^{136} \mathrm{Xe}$ using a 100-150 kg high-pressure gaseous xenon (HPGXe) time projection chamber. To amplify the ionization signal the detector uses electroluminicence (EL), that is, the emission of scintillation light after atom excitation by a charge accelerated by a moderately large (no charge gain) electric field. Electroluminescence is a linear process with a large gain that allows to obtain both good energy resolution and event topological information for background rejection.

Compared with the other two Xenon experiment, EXO and KamLAND-ZEN, NEXT displays a much better energy resolution, which can be as good as $0.5 \%$ at $Q_{\beta \beta}$ (to compare with $\sim 4 \%$ by EXO and $10 \%$ by KamLAND-ZEN), and the ability to use the topological information of the event to characterize the signal.

\subsection{Detection process}

The detection process is as follows. A charged particle propagating in the HPXe losses its energy through ionization and excitation of xenon atoms. The de-excitations of the atoms emits VUV light $(\sim 175 \mathrm{~nm})$ that is read using photodetectors behind a transparent cathode. Such fast signal, called $S_{1}$, provides the initial time of the event. A strong electric field is used to prevent the ionization electrons produced in the path of the charged particle to recombine with the positive ions (also generated for the primary charged particle), and also used to drift the negative charge towards the TPC anode. In the very last region of the TPC the charge enter inside a region of higher electric field exiting the Xenon atoms. Their de-excitation produces a huge number of VUV 
photons isotropically (EL process). The light propagated forward is detected with a set of small photosensors (MPPCs) of $1 \mathrm{~mm}^{2}$ placed in a matrix of $1 \mathrm{~cm}^{2}$ pitch giving a total coverage of $1 \%$, those photosensor are placed a few millimiters behind the anode and provide information about the event topology. In the other hand, the light propagated backwards are read with the same photodetectors used to read the primary light, with those photodetectors the energy information of the event is reconstructed with a resolution of $\sim 0.5 \%$ FWHM at $Q_{\beta \beta}$.

\section{NEXT-DEMO prototype}

This large prototype, operating at IFIC (Valencia, Spain) is a demonstrator of the full NEXT100 concept. It has two planes of photodetectors, one at the cathode and one at the anode, a long drift region $(30 \mathrm{~cm})$ and a field cage capable to withstand large voltages. The design operative pressure is 10 bar. The vessel is a stainless steel cylinder, $60 \mathrm{~cm}$ long and $30 \mathrm{~cm}$ diameter. The fiducial volume is defined by a hexagonal light-tube made of PTFE reflector panels, $160 \mathrm{~mm}$ across the diagonal and $300 \mathrm{~mm}$ drift region. The electroluminescence region is made of two parallel grids separated by $5 \mathrm{~mm}$. The maximum designed drift field is $1 \mathrm{kV} / \mathrm{cm}$ and the maximum electroluminescence field is $40 \mathrm{kV} / \mathrm{cm}$.

\subsection{Energy Function}

The energy function is performed with a PMTs plane behind the cathode that reads both $\mathrm{S} 1$ and $\mathrm{S} 2$.

\subsubsection{Geometrical effects:}

Z-position dependence due to attachment is easy corrected with an exponential function. XY position of the event is calculated using barycenter. A $2 \mathrm{D}$ profile that shows the differences in light collected as a function of the position is generated. The profile is then used to correct the response in the PMTs. The correction is applied iteratively until no spacial effects are observed.

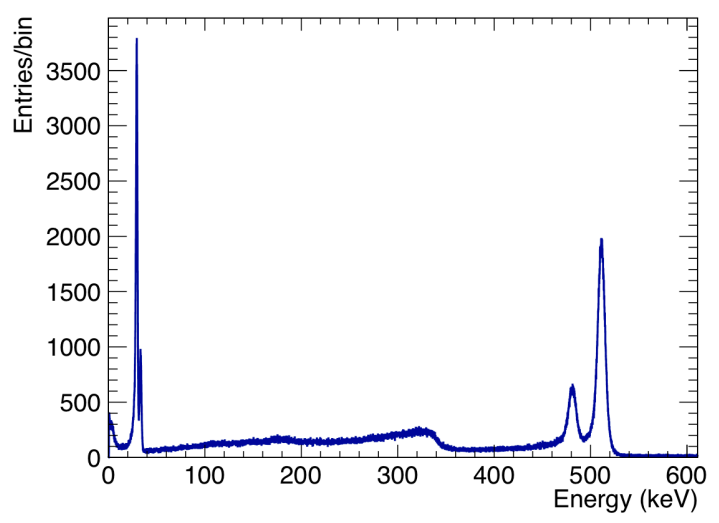

Figure 1: Energy spectrum after spatial corrections (left) and detail of the photoelectric peak with fit values (right).

A resolution of $1.92 \%$ FWHM at $511 \mathrm{keV}$ has been achieved. Extrapolation to $Q_{\beta \beta}$ gives $0.87 \%$ FWHM [5]].

\subsection{Topological Signature}

The topological reconstruccion of the events is realized using SiPMs in a rectangular array. The SiPMs are placed at $2 \mathrm{~mm}$ from the EL production area. The sensors are not sensitive to VUV light produced by the Xe so coating with a WLS chemical (tetraphenyl butadiene) is necessary to detect light.

A first approximation of the event topology can be made by subdividing the charge in time. This allows for a deeper understanding of an event's $\mathrm{z}$ structure and is the starting point for track reconstruction. For the first analysis, slices of $4 \mu \mathrm{s}$ width were used, as this width is comparable 

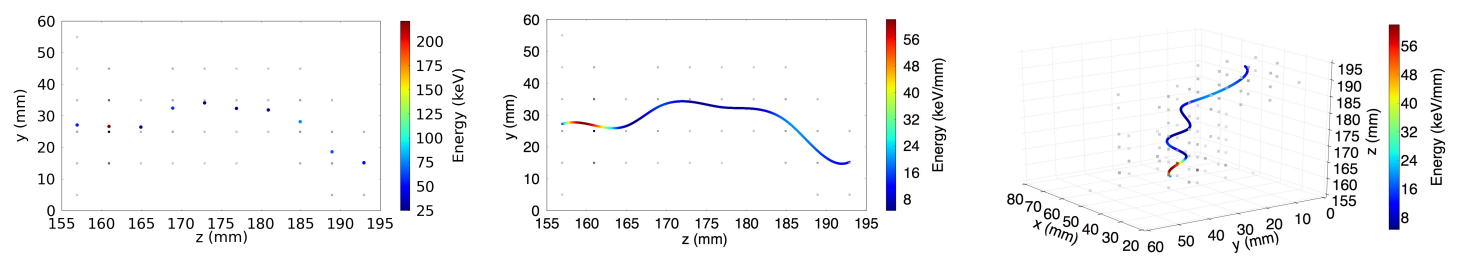

Figure 2: Example of ${ }^{137} \mathrm{Cs}$ track reconstruction: The charge of the different SiPMs is split into slices of $4 \mathrm{~mm}$ width in $z$. One point is calculated for each slice using the barycenter method and the energy of the points is then associated with the measurement made in the cathode (left). A cubic spline is used to interconect the different points; YZ projection (center) 3D (right).

to the time an electron needs to cross the EL region $(3 \mathrm{~s})$. In addition, the charge collected in a $4 \mathrm{~s}$ width window is sufficient to achieve a reliable xy reconstruction. Each time slice, while having a single $\mathrm{z}$ coordinate, can be comprised of multiple $\mathrm{x}, \mathrm{y}$ points. In this preliminary analysis a single xy point is reconstruced per slice and those events exhibiting slices with more than one isolated charge deposit in the tracking plane are excluded from analysis until a more sophisticated clustering algorithm is developed. The $x y$ position of a slice is reconstructed using its barycenter. This position then has the energy recorded in the cathode for the same time interval associated with it so that the event $\mathrm{dE} / \mathrm{dz}$ can be studied. The energy and position information are then used to calculate a cubic spline between the individual points in order to obtain a finer description of the path. Figure $\square$ illustrates the method applied to the reconstruction of a ${ }^{137} \mathrm{Cs}$ photoelectric event [四].

\section{Acknoledgments}

This work was supported by the Ministerio de Economía y Competitividad of Spain under grants CONSOLIDER-Ingenio 2010 CSD2008-0037 (CUP) and FPA2009-13697-C04-04. the Director, Office of Science, Office of Basic Energy Sciences, of the US Department of Energy under contract no. DE-AC02-05CH11231; and the Portuguese FCT and FEDER through the program COMPETE, project PTDC/FIS/103860/2008.

\section{References}

[1] M. C. Gonzalez-Garcia and M. Maltoni, Phenomenology with Massive Neutrinos, Phys. Rept. 460 (2008) 1 [arXiv:0704.1800 [hep-ph]].

[2] S. R. Elliott and P. Vogel, Ann. Rev. Nucl. Part. Sci. 52 (2002) 115 [hep-ph/0202264].

[3] F. T. Avignone, III, S. R. Elliott and J. Engel, Double Beta Decay, Majorana Neutrinos, and Neutrino Mass, Rev. Mod. Phys. 80 (2008) 481 [arXiv:0708.1033 [nucl-ex]].

[4] V. Alvarez et al. [NEXT Collaboration], NEXT-100 Technical Design Report (TDR): Executive Summary, JINST 7 (2012) T06001 [arXiv:1202.0721 [physics.ins-det]].

[5] V. Alvarez et al. [NEXT Collaboration], Initial results of NEXT-DEMO, a large-scale prototype of the NEXT-100 experiment, JINST 8 (2013) P04002 [arXiv:1211.4838 [physics.ins-det]].

[6] Álvarez, V. and others, Operation and first results of the NEXT-DEMO prototype using a silicon photomultiplier tracking array, arXiv:1306.0471 [physics.ins-det]. 